\title{
Review
}

\section{The Emergence of a New Conceptual Framework for Alzheimer's Disease}

\author{
Bruno Dubois* \\ Centre des Maladies Cognitives et Comportementales (IM2A), Institut du Cerveau et de la Moelle \\ épinière (ICM), UMR-S975, AP-HP, Salpêtrière Hospital, Pierre et Marie Curie University, Paris, France
}

Accepted 17 August 2017

\begin{abstract}
The New Criteria for the diagnosis of Alzheimer's disease (AD), published by a group of experts in 2007, have resulted in a revolution in the comprehension of the disease. Before 2007, the diagnosis of AD dementia was done through a process of exclusion: it was considered in the case of patients with a dementia syndrome without identified etiologies. This traditional algorithm had three major limitations that penalize the disease: 1) a low accuracy of the performance which may share responsibility for negative results in clinical trials; 2) a late identification of the patients only when they reach the threshold of dementia which may delay the activation of optimal care; and last but not least, 3) an absence of clear recognition of AD as a disease because of the lack of specific arguments for its identification. Since 2007, the disease has gained a clear definition based on positive evidence: a specific clinical phenotype (the amnestic syndrome of the hippocampal type) and the presence of biomarkers, considered as a biological signature of the disease. Thanks to these positive arguments, AD is a clinically and biologically well-delineated disease, no longer defined as "probable". It is now possible to certify that a given patient has or does not have the disease. Like diabetes, cancer, hyperthyroidism or any other disorder, AD has now a clear definition with well-defined borders. The disease has entered the world of medicine with identified diseases with a biological fingerprint. This is the story of this adventure that we will present now.
\end{abstract}

Keywords: Alzheimer's disease, biomarkers, criteria, diagnosis, preclinical stage, prodromal stage

\section{INTRODUCTION}

Based on the classical diagnostic criteria proposed by the National Institute of Neurological and Communicative Disorders and Stroke and the Alzheimer's Disease and Related Disorders Association (NINCDS-ADRDA) in 1984, the clinical diagnosis of Alzheimer's disease (AD) was initially considered within a 2-step procedure with an initial identification of a dementia syndrome and then the exclusion of other possible etiologies of dementia with blood/cerebrospinal fluid (CSF) investigations

\footnotetext{
*Correspondence to: Bruno Dubois, IM2A, Pavillon François Lhermitte, Hôpital Salpêtrière, 47 Bd de l'Hôpital, 75013 Paris, France. Tel.: +331421675 02; E-mail: bruno.dubois@aphp.fr.
}

to rule out infectious, inflammatory, or metabolic diseases and with brain neuroimaging [computed tomography (CT) scan or magnetic resonance imaging (MRI)] for excluding small vessel diseases, strategic lacunar infarcts, large vessel infarcts and/or cerebral hemorrhages, brains tumors, hydrocephalus, etc. This was mainly an exclusionary process. Based on these criteria, the clinical diagnosis of AD could not be certified ("probable" AD) and a definite diagnosis needed a histological confirmation based on cerebral biopsy or postmortem examination [1].

In this context, the emergence of a new conceptual framework for AD was progressive and relied on different successive steps that are presented in a chronological fashion. 


\section{DISENTANGLING THE MEMORY PROFILES OF DEGENERATIVE DISORDERS}

Having worked for years on the cognitive and behavioral changes associated with neurodegenerative disorders involving primarily subcortical structures and basal ganglia (Parkinson's disease, progressive supranuclear palsy, or Huntington disease), we have conceptualized that these disorders are mainly characterized by a severe executive dysfunction [2]. The frontal dysfunction has a significant impact in various domains in these patients: increased apathy, slowing of central processing time, decreased fluency, lack of flexibility, difficulties in conceptualization and problems solving ability, and memory deficits [3]. Long-term memory is apparently affected in subcortico-frontal dementias as shown by the low performance in word-list recall [4]. However, when encoding of information is controlled in the initial stage of memory processing using semantic category cues and when recall is facilitated with the same cues as with tests such as the Free and Cued Selective Reminding Test (FCSRT) [5], patients' recall performance is dramatically improved. The preserved ability to retrieve all the items indicates that there was no genuine amnesia in these diseases [4].

This subcortico-frontal pattern of memory deficit is strikingly different from that of patients with AD. In $\mathrm{AD}$, recall performance is not normalized by cueing, indicating the presence of a storage deficit. This finding led us to propose a functional "mediotemporal versus frontal dissociation" to explain the different patterns of memory disorders observed in neurodegenerative diseases [3]. The frontal lobe component mediates the internally generated strategy to guide memory search, which explains the low performance in free recall in patients with frontal lobe dysfunctions. By contrast, the medial temporal/hippocampal component is predominantly impaired in $\mathrm{AD}$ and is responsible for encoding deficits, loss of information after delay, low effect of cueing on recall, and high number of extra-list intrusion and false positives in recognition. Therefore, based on these initial studies, we postulated that it is possible to isolate a specific pattern of memory profile that is related to hippocampal damage [6]. In line with this hypothesis, we found that a decrease in the total recall score (the sum of free and cued recall) and in the index of sensitivity to semantic cueing (the percentage of cues that activate the retrieval of correct items that were not spontaneously recalled) were significantly related to $\mathrm{AD}$ and that both are good markers of the disease [7].

To summarize, if a poor free recall is the rule in any type of brain damage with memory disorders, a low performance in total recall despite retrieval facilitation is a marker of medio temporal/hippocampal dysfunction and therefore of AD. This "amnestic syndrome of the hippocampal type" successfully differentiates patients with $\mathrm{AD}$ from any other memory disorders that are seen in depression, frontal lobe dysfunction, subcortical dementias, or vascular dementias where a low free recall performance is generally normalized (or quasi normalized) by cueing [8].

\section{AD IS A RATHER HOMOGENEOUS DISEASE WITH A STARTING POINT IN MEDIAL TEMPORAL LOBE STRUCTURES}

In parallel, the diagnosis accuracy of AD improved significantly in the last decades because of the characterization of new dementias through specific criteria, including the primary progressive aphasias [9], semantic dementia [10], cortico-basal degeneration [11], posterior-cortical atrophy [12], and Lewy body dementia [13].

The individualization of these new diseases, which were previously confused with $\mathrm{AD}$, has consequently decreased its apparent heterogeneity. At the same time, postmortem studies of $\mathrm{AD}$ patients provide evidence of a rather specific pattern of cerebral neuronal lesions, which appear to begin within the medial temporal lobe structures (entorhinal cortex, hippocampal formations, para-hippocampal gyrus) [14], structures known to be critical for long-term episodic memory. According to the Braak stages, the initial media-temporal lobe involvement is followed by a progressive diffusion of neuronal lesions to the neocortical areas [15].

\section{AD IS A PROGRESSIVE AMNESTIC DEMENTIA}

According to this model, we have proposed that episodic memory deficit is an early and reliable neuropsychological marker of $\mathrm{AD}$ [8]. In a follow-up study of 251 patients with mild cognitive impairment (MCI), an amnestic syndrome of the hippocampal type evidenced by the FCSRT, was already present and severe. Moreover, it was by far the best predictor for a further progression to $\mathrm{AD}$ dementia within the 
next 3 years among all the standardized neuropsychological battery-which included in addition the Benton visual retention test for visual memory, the Deno 100 and verbal fluency for language, the serial digit ordering test and the double task of Badley for working memory, the similarities of the Wechsler Adult Intelligence Scale (WAIS) for conceptual elaboration, the Stroop task, the Trail Making Test, and the WAIS digit symbol test for executive functions [16]. This pattern was also shown to correlate with hippocampal atrophy [17] and grey matter loss of the medial temporal lobe [18]. In addition, it predicts with high accuracy the presence of AD pathology determined in vivo by CSF changes [18, 19] even at a prodromal stage of the disease [20]. Based on these evidence, we proposed AD as characterized by a rather homogeneous clinical presentation, which can be divided into two mains stages: 1) the first stage consists of a progressive and rather isolated amnestic syndrome in relation in the early involvement of the medio-temporal structures; 2 ) the second is characterized by the addition and the development of cognitive symptoms in the domain of executive (conceptualization, judgment, problem solving) and instrumental (language, praxis, face or object recognition) functions and of psycho-behavioral changes, due to the progressive invading of neocortical areas with neuronal lesions.

\section{IDENTIFICATION OF A PRODROMAL STAGE OF AD}

AD pathology is well advanced by the time patients present with their first cognitive symptoms, even though they may not meet current criteria for dementia. Considering AD only at a dementia stage is detrimental to care for patients affected by the disease. There is no reason to link the diagnosis of a disease with a certain threshold of severity and to exclude a large number of patients who are not yet expressing a full-blown dementia from diagnosis and treatment. Early intervention for drug development may be important for effective treatment and there is no justification to anchor the diagnosis of $\mathrm{AD}$ to a dementia syndrome. For these reasons, we had already considered in 2000 that the "concept of prodromal AD was more useful than MCI" [21]. MCI is a heterogeneous condition with various underlying etiologies. For the clinician and for the patient, the problem is not diagnosing the syndrome (MCI) but rather detecting the underlying disease (prodromal
AD) responsible for the syndrome. The presence of the specific memory pattern, the amnestic of the hippocampal type, even at a predementia stage of the disease, makes it possible to identify, or at least to suspect, AD at a prodromal stage. However, affirming the diagnosis at this stage might be difficult and the consequence of a false diagnosis is very serious. In this context, the discovery of biomarkers of the disease was very timely.

\section{THE ADDED VALUE OF BIOMARKERS}

The clinical diagnosis, suggested by a specific phenotype (pattern of memory disorders either isolated or associated with cognitive/behavioral changes), can now be confirmed by the presence of positive biomarkers. For the last decades since the NINCDS-ADRDA criteria were published, great progress has been made with the identification of the AD-associated structural molecular brain changes and their biochemical footprints. MRI enables the measure of medial temporal lobe structures [22]. Positron emission tomography (PET) with fluorodeoxyglucose (FDG) may evidence a temporoparietal hypometabolism associated with $\mathrm{AD}$ even in the early stages [23]. Analysis for CSF can detect the key molecular pathological features of AD [24] as well as PET with amyloid ligands [25], even the prodromal stage of the disease. The availability of specific biomarkers of $\mathrm{AD}$ pathology in vivo supports a major change in the conceptualization and diagnosis of the disease, moving the definition of AD from a clinico-pathological entity to a clinico-biological entity. As biomarkers can be considered as surrogate markers of the histopathological changes, the clinical diagnosis can now be established in vivo at any stage of the disease. Reference to dementia may no longer be needed for diagnosing $\mathrm{AD}$. AD should be now considered as a continuum, and it was timely to propose a new set that applies at any stage of the disease.

\section{REVISING THE NINCDS-ADRDA CRITERIA}

Considering these advances in clinical phenotype and biomarkers of the disease, providing positive evidence for the diagnosis, we convened with Philip Scheltens and Howard Feldman a working group with 15 international dementia experts in Firenze in 2005 to develop a diagnostic framework for AD that would 
Table 1

The new diagnostic approach

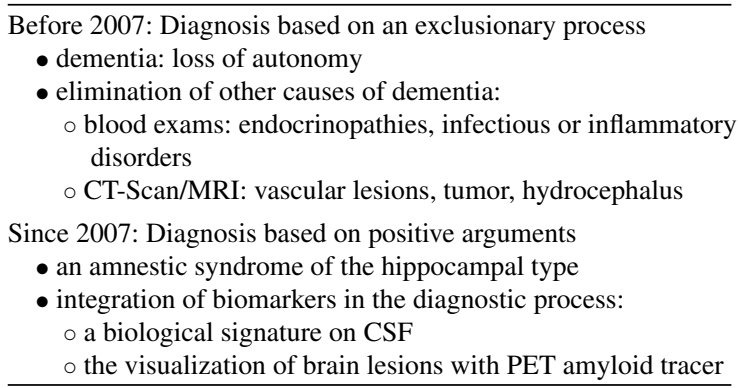

include the prodromal stages and the integration of biomarkers and to define the future goals and steps for the validation of such a framework. The workshop was at the origin of a 2007 Lancet Neurology paper [26] on the new research criteria for typical AD. These new criteria proposed that AD can now be defined in vivo with a diagnostic algorithm that is centered on a clinical core of early and significant episodic memory impairment and then requires supporting biomarkers among structural neuroimaging with MRI, molecular neuroimaging with PET, and CSF analysis of amyloid- $\beta(A \beta)$ or tau proteins (see Table 1). AD is now a clinico-biological entity, which can be identified in vivo and encompasses the whole spectrum of its clinical course. At the time where we published the New Criteria, the respective contribution and the weight of each of biomarker in terms of diagnosis accuracy was not known and this is the reason for the subsequent articles that we published on this matter.

\section{THE NEW LEXICON FOR AD}

The new conceptual framework of AD suggests redefining a common lexicon concerning $\mathrm{AD}$ and related entities [27].

\section{Alzheimer's disease}

AD now refers to the whole spectrum of the clinical phase of the disease and is not restricted to the dementia syndrome. The diagnosis requires the evidence of an amnestic syndrome of the hippocampal type (defined by a free recall deficit that is not normalized by cueing) and the presence of pathophysiological markers of AD. Distinguishing two different stages may still be meaningful: a prodromal and a dementia phase. However, the transition between the two states may be arbitrary because the underlying disease is a continuous process. Individual clinician's experience will impact significantly on the threshold of detection of the transition to $\mathrm{AD}$.

\section{AD dementia}

This term refers to the phase of AD during which cognitive symptoms are sufficiently severe to interfere with social functioning and instrumental activities of daily living, a threshold that is considered to define dementia in association with changes in episodic memory and in at least one other cognitive domain.

\section{Prodromal $A D$}

This refers to the early symptomatic, predementia phase of the disease, characterized by a specific clinical phenotype of the amnestic syndrome of the hippocampal type with positive pathophysiological biomarkers. The memory disorders can be isolated or associated with other cognitive or behavioral changes that are not severe enough to interfere significantly with activities of daily living. The term of prodromal $\mathrm{AD}$ might disappear in the future if $\mathrm{AD}$ is considered to encompass both the predementia and dementia stages.

\section{Typical AD}

This term refers to the most common clinical phenotype of $\mathrm{AD}$ (more than $85 \%$ of the cases), characterized by an early significant and progressive episodic memory deficit that remains dominant in the later stages of the disease, and is followed by or associated with other cognitive impairments (executive dysfunction, language, praxis, and complex visual processing impairments) and neuropsychiatric changes. The diagnosis is further supported by one or more in-vivo positive biomarkers of $\mathrm{AD}$ pathology.

\section{Atypical AD}

This term refers to the less common clinical phenotypes that may occur with $\mathrm{AD}$ pathology: logopenic aphasia, posterior cortical atrophy, and frontal variant of $\mathrm{AD}$. In the presence of one of these clinical presentations, the diagnosis of AD is supported by in vivo evidence of pathophysiological biomarkers of $\mathrm{AD}$. 


\section{Mixed $A D$}

This term refers to patients who fulfill the diagnostic criteria for typical $\mathrm{AD}$ and additionally present with clinical and brain imaging/biological evidence of other comorbid disorders such as cerebrovascular disease or Lewy body disease.

\section{Preclinical $A D$}

This term refers to the long asymptomatic stage between the earliest pathogenic events/brain lesions of $\mathrm{AD}$ and the first appearance of specific cognitive changes. Two preclinical states can be isolated in vivo:

Asymptomatic at-risk for AD: Cognitively normal individuals with biomarker evidence of AD pathology. In the absence of knowledge about the value of these biological changes to predict the further development of the disease, they should be referred to as an "at-risk for AD".

Presymptomatic AD: Cognitively normal individuals sharing an autosomal dominant monogenic AD mutation. Because of the full penetrance of the mutations, they will develop a clinical $\mathrm{AD}$ if they live long enough.

\section{Biomarkers of $A D$}

The International Working Group (IWG) has proposed to divide biomarkers into pathophysiological and topographical markers [27] (see Table 2).

Pathophysiological markers identify AD pathology. They target the two etiological degenerative processes that characterize AD pathology: amyloidosis and tauopathy. They are markers of diagnosis. They include CSF changes with decreased $A \beta$ and

Table 2

Definition of AD biomarkers

\begin{tabular}{l}
\hline Diagnostic marker \\
- Pathophysiological marker \\
- Reflects in-vivo pathology \\
- Is present at all stages of the disease \\
- Observable even in the asymptomatic state \\
- Might not be correlated with clinical severity \\
- Indicated for inclusion in protocols of clinical trials \\
Progression marker \\
- Topographical or downstream marker \\
- Poor disease specificity \\
- Indicates clinical severity (staging marker) \\
- Might not be present in early stages \\
- Quantifies time to disease milestones \\
- Indicated for disease progression \\
\hline
\end{tabular}

increased total- and phospho-tau levels, as well as increased brain retention of amyloid radioligand observed with PET. Total-tau level reflects the intensity of neural degeneration in the brain; phospho-tau levels the tangle pathology; and $A \beta_{1-42}$ levels the amyloid burden [28].

Topographical markers evaluate less specific and downstream brain changes that result from $\mathrm{AD}$ pathology. They assess changes over time and are therefore markers of progression more targeted at assessing changes over time and predicting outcomes. They include medial temporal lobe atrophy, particularly of the hippocampus [29] and reduced glucose metabolism in temporo-parietal regions on FDG PET [30].

\section{Mild cognitive impairment}

This diagnostic label is applied if there is no disease to which MCI can be attributed. It remains a term of exclusion for individuals who do not meet the proposed new criteria for $\mathrm{AD}$, in that they deviate from the clinicobiological phenotype of prodromal $\mathrm{AD}$ because they have memory symptoms that are not characteristic of $\mathrm{AD}$ or because they are biomarker negative.

\section{UPDATING OF THE NEW CRITERIA}

A new position paper was published some years after to improve the diagnostic framework [31]. Several refinements were proposed that have been made possible because rapid progress in the field in the past 4 years has improved our characterization of clinical phenotypes and the expression of disease captured by in vivo biomarkers of AD pathology. We propose that pathophysiological biomarkers indicating the specific presence of tau pathology (CSF or PET tau) and amyloid pathology (CSF or PET amyloid) have the necessary specificity for a diagnosis of $\mathrm{AD}$ at any point on the disease continuum. Downstream topographical markers of brain regional structural and metabolic changes have insufficient pathological specificity and are therefore now removed from the IWG diagnostic algorithm. Emphasis was also made on atypical forms of $\mathrm{AD}$, which represent an estimated $6-14 \%$ of cases [32]. Each of these atypical forms of $\mathrm{AD}$ presents with a relative preservation of memory plus a recognizable (or characteristic) phenotype that might be accompanied by topographical evidence of brain damage (regional atrophy or hypometabolism) in related regions. It is now possible 
Table 3

Added-value of the IWG-2 criteria

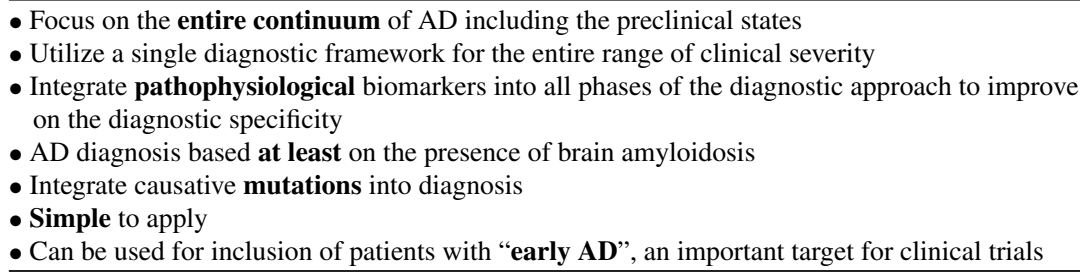

to propose more precise definitions for atypical AD presentations, including a posterior variant of $\mathrm{AD}$, a logopenic variant of $\mathrm{AD}$, and a frontal variant of $\mathrm{AD}$.

On the basis of these refinements, the diagnosis of $\mathrm{AD}$ can be simplified, requiring the presence of an appropriate clinical AD phenotype (typical or atypical) and a pathophysiological biomarker consistent with the presence of AD pathology (IWG-2 criteria, see Table 3). Thanks to the pathophysiological biomarkers, the criteria may apply for any stage of the disease (preclinical, prodromal, and dementia stages) considering the principle according to which: "One disease, one set of criteria". We propose that downstream topographical biomarkers of the disease, such as volumetric MRI and fluorodeoxyglucose PET, might better serve in the measurement and monitoring of the course of disease. This paper also elaborates on the specific diagnostic criteria for atypical forms of $\mathrm{AD}$, for mixed $\mathrm{AD}$, and for the preclinical states of $\mathrm{AD}$.

\section{DEFINING THE PRECLINICAL STATE OF AD}

During the past decade, the conceptual shift occurred in the field of $\mathrm{AD}$ considering the disease as a continuum. Thanks to evolving biomarker research and substantial discoveries, it is now possible to identify the disease even at the preclinical stage before the occurrence of the first clinical symptoms. This preclinical stage of $\mathrm{AD}$ has become a major research focus as the field postulates that early intervention may offer the best chance of therapeutic success. However, very little evidence is established on this "silent" stage of the disease and its definition varies from one study to the next [33]. A clarification was needed about the definitions and lexicon, the limits, the natural history, the markers of progression, and the ethical consequence of detected the disease at this asymptomatic stage. For these reasons, a consensus paper was published, addressing all these issues by providing evidence and practical recommendation [34]. For instance, a relationship between asymptomatic at risk and preclinical $\mathrm{AD}$ is only a matter of level of risk. Based on this high/low risk dichotomy, "preclinical AD" should be considered when the risk for a further progression to a clinical $\mathrm{AD}$ is particularly high (e.g., both $\mathrm{A} \beta$ and tau markers beyond pathological thresholds) and "asymptomatic at risk for AD" should be considered when the evolution to a clinical AD is less slightly or still needs to be determined (when the biomarkers pattern is insufficient with only one pathophysiological marker being abnormal) (see Table 4 and Fig. 1). The risk of progression to a clinical $\mathrm{AD}$ is the expression of a complex algorithm where the presence of $\mathrm{AD}$ brain lesions plays a key role and additional positive and/or negative factors need to be considered. If the sequential appearance of existing biomarkers and the polygenic and environmental protective/risk factors is considered, it should be possible, at some points, to predict the risk spectrum of a given individual and the putative time to the onset of a clinical disease, that is, to determine his conditions along a risk spectrum, ranging from negligible risk to immediate risk. Today, several genetic and lifestyle factors have been identified that may delay the onset of a clinical AD. After identifying subjects at risk for $\mathrm{AD}$, based on the presence of specific biological predictors, the main issue will be to detect those with the highest likelihood to progress to definite clinical AD in the forthcoming months to years. The identification of markers, able to detect disease progression in a relative short time frame, before the onset of clinical symptoms becomes of crucial importance. To date, evidence from studies on blood-based biomarkers indicates a limited value for the characterization of preclinical stage of AD. Therefore, in the absence of definitive answers concerning the algorithm of progression to a clinical disease, we have considered that the knowledge of biomarkers status should not be disclosed at a preclinical stage. 
Table 4

Consensus on two different states
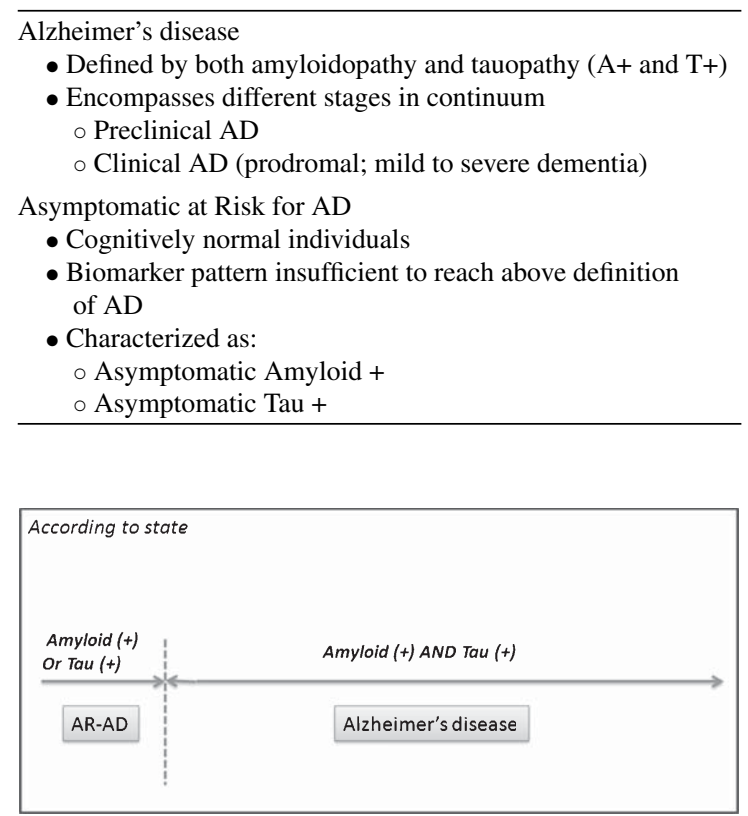

Fig. 1. Definition of AD and of asymptomatic at risk. A consensus on the definition of AD based on specific biomarkers was reached (Dubois et al. [34]), which works for any stage of the disease, even for the preclinical stage of the disease. Asymptomatic at risk for $\mathrm{AD}$ are identified when the biomarker pattern is insufficient to reach the above definition of $\mathrm{AD}$.

To conclude, it is noteworthy that significant progress was made in the diagnostic accuracy of $\mathrm{AD}$ during these last years. However, it is unfortunate that this is not yet translated into new perspectives for both symptomatic and disease modifying therapies.

\section{DISCLOSURE STATEMENT}

The author's disclosure is available online (http://jalz.com/manuscript-disclosures/17-0536r1).

\section{REFERENCES}

[1] McKhann G, Drachman D, Folstein M, Katzman R, Price D, Stadlan EM (1984) Clinical diagnosis of Alzheimer's disease: Report of the NINCDS-ADRDA Work Group under the auspices of Department of Health and Human Services Task Force on Alzheimer's Disease. Neurology 34, 939-944.

[2] Pillon B, Deweer B, Michon A, Malapani C, Agid Y, Dubois B (1994) Are explicit memory disorders of progressive supranuclear palsy related to damage to striatofrontal circuits? Comparison with Alzheimer's, Parkinson's, and Huntington's diseases. Neurology 44, 1264-1270.

[3] Dubois B, Andrade K, Levy R (2008) Executive dysfunction and neuropsychological testing. Handb Clin Neurol 89, 35-52.
[4] Pillon B, Deweer B, Agid Y, Dubois B (1993) Explicit memory in Alzheimer's, Huntington's, and Parkinson's diseases. Arch Neurol 50, 374-379.

[5] Grober E, Buschke H, Crystal H, Bang S, Dresner R (1988) Screening for dementia by memory testing. Neurology $\mathbf{3 8}$, 900-903.

[6] Deweer B, Lehericy S, Pillon B, Baulac M, Chiras J, Marsault C, Agid Y, Dubois B (1995) Memory disorders in probable Alzheimer's disease: The role of hippocampal atrophy as shown with MRI. J Neurol Neurosurg Psychiatry 58, 590-597.

[7] Tounsi H, Deweer B, Ergis AM, Van der Linden M, Pillon B, Michon A, Dubois B (1999) Sensitivity to semantic cuing: An index of episodic memory dysfunction in early Alzheimer disease. Alzheimer Dis Assoc Disord 13, 38-46.

[8] Dubois B, Albert ML (2004) Amnestic MCI or prodromal Alzheimer's disease? Lancet Neurol 3, 246-248.

[9] Mesulam MM (1987) Primary progressive aphasiadifferentiation from Alzheimer's disease. Ann Neurol 22, 533-534.

[10] Hodges JR, Patterson K, Oxbury S, Funnell E (1992) Semantic dementia. Progressive fluent aphasia with temporal lobe atrophy. Brain 115(Pt 6), 1783-1806.

[11] Gibb WR, Luthert PJ, Marsden CD (1989) Corticobasal degeneration. Brain 112(Pt 5), 1171-1192.

[12] Benson DF, Davis RJ, Snyder BD (1988) Posterior cortical atrophy. Arch Neurol 45, 789-793.

[13] McKeith IG, Galasko D, Wilcock GK, Byrne EJ (1995) Lewy body dementia-diagnosis and treatment. Br J Psychiatry 167, 709-717.

[14] Delacourte A, David JP, Sergeant N, Buée L, Wattez A, Vermersch P, Ghozali F, Fallet-Bianco C, Pasquier F, Lebert F, Petit H, Di Menza C (1999) The biochemical pathway of neurofibrillary degeneration in aging and Alzheimer's disease. Neurology 52, 1158-1165.

[15] Braak H, Braak E (1991) Neuropathological stageing of Alzheimer-related changes. Acta Neuropathol 82, 239-259.

[16] Sarazin M, Berr C, De Rotrou J, Fabrigoule C, Pasquier F, Legrain S, Michel B, Puel M, Volteau M, Touchon J, Verny M, Dubois B (2007) Amnestic syndrome of the medial temporal type identifies prodromal AD: A longitudinal study. Neurology 69, 1859-1867.

[17] Sarazin M, Chauvire V, Gerardin E, Colliot O, Kinkingnéhun S, de Souza LC, Hugonot-Diener L, Garnero L, Lehéricy S, Chupin M, Dubois B (2010) The amnestic syndrome of hippocampal type in Alzheimer's disease: An MRI study. J Alzheimers Dis 22, 285-294.

[18] Rami L, Sole-Padulles C, Fortea J, Bosch B, Lladó A, Antonell A, Olives J, Castellví M, Bartres-Faz D, SánchezValle R, Molinuevo JL (2012) Applying the new research diagnostic criteria: MRI findings and neuropsychological correlations of prodromal AD. Int J Geriatr Psychiatry 27, 127-134.

[19] Wagner M, Wolf S, Reischies FM, Daerr M, Wolfsgruber S, Jessen F, Popp J, Maier W, Hüll M, Frölich L, Hampel H, Perneczky R, Peters O, Jahn H, Luckhaus C, Gertz HJ, Schröder J, Pantel J, Lewczuk P, Kornhuber J, Wiltfang J (2012) Biomarker validation of a cued recall memory deficit in prodromal Alzheimer disease. Neurology 78, 379-386.

[20] Derby CA, Burns LC, Wang C, Katz MJ, Zimmerman ME, L'italien G, Guo Z, Berman RM, Lipton RB (2013) Screening for predementia AD: Time-dependent operating characteristics of episodic memory tests. Neurology $\mathbf{8 0}$, 1307-1314. 
[21] Dubois B (2000) 'Prodromal Alzheimer's disease': A more useful concept than mild cognitive impairment? Curr Opin Neurol 13, 367-369.

[22] Lehericy S, Baulac M, Chiras J, Pierot L, Martin N, Pillon B, Deweer B, Dubois B, Marsault C (1994) Amygdalohippocampal MR volume measurements in the early stages of Alzheimer disease. Am J Neuroradiol 15, 929-937.

[23] Silverman DH, Gambhir SS, Huang HW, Schwimmer J, Kim S, Small GW, Chodosh J, Czernin J, Phelps ME (2002) Evaluating early dementia with and without assessment of regional cerebral metabolism by PET: A comparison of predicted costs and benefits. J Nucl Med 43, 253-266.

[24] Hansson O, Zetterberg H, Buchhave P, Londos E, Blennow K, Minthon L (2006) Association between CSF biomarkers and incipient Alzheimer's disease in patients with mild cognitive impairment: A follow-up study. Lancet Neurol 5, 228-234.

[25] Klunk WE, Engler H, Nordberg A, Wang Y, Blomqvist G, Holt DP, Bergström M, Savitcheva I, Huang GF, Estrada S, Ausén B, Debnath ML, Barletta J, Price JC, Sandell J, Lopresti BJ, Wall A, Koivisto P, Antoni G, Mathis CA, Långström B (2004) Imaging brain amyloid in Alzheimer's disease with Pittsburgh Compound-B. Ann Neurol 55, 306-319.

[26] Dubois B, Feldman H, Jacova C, DeKosky ST, BarbergerGateau P, Cummings J, Delacourte A, Galasko D, Gauthier S, Jicha G, Meguro K, O’Brien J, Pasquier F, Robert P, Rossor M, Salloway S, Stern Y, Visser PJ., Scheltens P (2007) Research criteria for the diagnosis of Alzheimer's disease: Revising the NINCDS-ADRDA criteria. Lancet Neurol 6, 734-746.

[27] Dubois B, Feldman HH, Jacova C, Cummings JL, Dekosky ST, Barberger-Gateau P, Delacourte A, Frisoni G, Fox NC, Galasko D, Gauthier S, Hampel H, Jicha GA, Meguro K, O'Brien J, Pasquier F, Robert P, Rossor M, Salloway S, Sarazin M, de Souza LC, Stern Y, Visser PJ, Scheltens P (2010) Revising the definition of Alzheimer's disease: A new lexicon. Lancet Neurol 9, 1118-1127.

[28] Blennow K, Hampel H, Weiner M, Zetterberg H (2010) Cerebrospinal fluid and plasma biomarkers in Alzheimer disease. Nat Rev Neurol 6, 131-144.
[29] Sabuncu MR, Desikan RS, Sepulcre J, Yeo BT, Liu H, Schmansky NJ, Reuter M, Weiner MW, Buckner RL, Sperling RA, Fischl B, Alzheimer's Disease Neuroimaging Initiative (2011) The dynamics of cortical and hippocampal atrophy in Alzheimer disease. Alzheimer's Disease Neuroimaging Initiative. Arch Neurol 68, 1040-1048.

[30] Mosconi L (2005) Brain glucose metabolism in the early and specific diagnosis of Alzheimer's disease. FDG-PET studies in MCI and AD. Eur J Nucl Med Mol Imaging 32, 486-510.

[31] Dubois B, Feldman HH, Jacova C, Hampel H, Molinuevo JL, Blennow K, DeKosky ST, Gauthier S, Selkoe D, Bateman R, Cappa S, Crutch S, Engelborghs S, Frisoni GB, Fox NC, Galasko D, Habert MO, Jicha GA, Nordberg A, Pasquier F, Rabinovici G, Robert P, Rowe C, Salloway S, Sarazin M, Epelbaum S, de Souza LC, Vellas B, Visser PJ, Schneider L, Stern Y, Scheltens P, Cummings JL (2014) Advancing research diagnostic criteria for Alzheimer's disease: The IWG-2 criteria. Lancet Neurol 13, 614-629.

[32] Galton CJ, Patterson K, Xuereb JH, Hodges JR (2000) Atypical and typical presentations of Alzheimer's disease: A clinical, neuropsychological, neuroimaging and pathological study of 13 cases. Brain 123, 484-498.

[33] Epelbaum S, Genthon R, Cavedo E, Habert MO, Lamari F, Gagliardi G, Lista S, Teichmann M, Bakardjian H, Hampel H, Dubois B (2017) Preclinical Alzheimer's disease: A systematic review of the cohorts underlying the concept. Alzheimers Dement 13, 454-467.

[34] Dubois B, Hampel H, Feldman H, Scheltens P, Aisen P, Andrieu S, Bakardjian H, Benali H, Bertram L, Blennow K, Broich K, Cavedo E, Crutch S, Dartigues JF, Duyckaerts C, Epelbaum S, Frisoni GB, Gauthier S, Genthon R, Gouw AA, Habert MO, Holtzman DM, Kivipelto M, Lista S, Molinuevo JL, O'Bryant SE, Rabinovici GD, Rowe C, Salloway S, Schneider LS, Sperling R, Teichmann M, Carrillo MC, Cummings J, Jack CR Jr, Proceedings of the Meeting of the International Working Group (IWG) and the American Alzheimer's Association on "The Preclinical State of AD”, July 23, 2015, Washington DC, USA (2016) Preclinical Alzheimer's disease: Definition, natural history, and diagnostic criteria. Alzheimers Dement 12, 292-323. 